\title{
THE PRODUCT OF TOTALLY NONMEAGRE SPACES
}

\author{
J. M. AARTS AND D. J. LUTZER ${ }^{1}$
}

\begin{abstract}
In this note we give an example of a separable, pseudo-complete metric space $X$ which is totally nonmeagre (= every closed subspace of $X$ is a Baire space) and yet whose square $X \times X$ is not totally nonmeagre.
\end{abstract}

1. Introduction. All spaces considered in this note are assumed to be regular and $T_{1}$. A space $X$ is totally nonmeagre if every closed subspace of $X$ is a Baire space, or, equivalently, if every nonempty closed subspace of $X$ has second category in itself [2]. The classical examples of Baire spacescomplete metric spaces, locally compact Hausdorff spaces, and (locally) Čech-complete spaces [4]—are all totally nonmeagre.

Oxtoby [6] has given an example which shows that the product $X \times Y$ of two Baire spaces need not be a Baire space. However, it is known that if the Baire space $X$ satisfies certain additional hypotheses, then $X \times Y$ is a Baire space for any Baire space $Y$. Two examples of such additional hypotheses are:

(A) that $X$ has a locally countable pseudo-base [6] (which is equivalent, for a metric space, to the existence of a dense open subspace which is locally separable-e.g., if $X$ is itself separable);

(B) that $X$ is pseudo-complete [1], [6] (which is equivalent, for a metric space, to the existence of a dense, completely metrizable subspacee.g., if $X$ contains a dense set of isolated points; all of the classical examples of Baire spaces are pseudo-complete).

The purpose of this note is twofold: first, to give an example of a totally nonmeagre metric space $X$ which is both separable and pseudo-complete and yet whose square $X \times X$ is not totally nonmeagre; and second, to suggest the following open question: if $X$ is compact Hausdorff and $Y$ is totally nonmeagre, must $X \times Y$ be totally nonmeagre?

2. The example. A separable metrizable space is totally imperfect if it has no dense-in-itself completely metrizable subspaces, or, equivalently, if it has no uncountable compact subspaces [5].

Received by the editors June 22, 1972.

AMS (MOS) subject classifications (1970). Primary 54E99; Secondary 54G15, 54H05.

Key words and phrases. Baire space, totally nonmeagre space, totally imperfect sets.

${ }^{1}$ Partially supported by NSF Grant GP 29401.

(c) American Mathematical Society 1973 
Lemma 1. Let $Y$ be separable, completely metrizable and dense-initself, and let $X \subset Y$. If $Y \backslash X$ is totally imperfect, then $X$ is totally nonmeagre.

Proof. Suppose $F \neq \varnothing$ is a closed subset of $X$ which can be written as $F=\bigcup_{n=1}^{\infty} F_{n}$ where each $F_{n}$ is closed and nowhere dense in $F$ (whence also in $X$ ). Let $H=\mathrm{Cl}_{Y}(F)$ and $H_{n}=\mathrm{Cl}_{Y}\left(F_{n}\right)$. Then $H$ is dense-in-itself, each $H_{n}$ is nowhere dense in $H$, and $\varnothing \neq H \backslash F \subset Y \backslash X$ because $F$, being of first category in itself, cannot be closed in the complete space $Y$. Therefore $K=H \backslash\left(\bigcup_{n=1}^{\infty} H_{n}\right)$ is a dense $G_{\delta}$-subset of $H$. Hence $K$ is dense-initself and completely metrizable [3]. But this is impossible since $K$ is a subset of the totally imperfect space $Y \backslash X$.

A well-known theorem of Bernstein states that any separable completely metrizable space $X$ which is dense-in-itself can be decomposed into two disjoint totally imperfect subsets $Y$ and $Z$ [5]. (In view of Lemma 1, both $Y$ and $Z$ are totally nonmeagre.) For our purposes we need a slightly modified version of Bernstein's theorem.

Throughout the rest of this section, let $R$ and $Q$ denote respectively the usual spaces of real and rational numbers. Let $R^{+}=\{x \in R: x \geqq 0\}$ and let $Q^{+}=Q \cap R^{+}$.

Lemma 2. There exist totally imperfect subsets $C$ and $D$ of $R^{+}$having $C \cap D=Q^{+}$and $C \cup D=R^{+}$.

Proof. Well order the collection of all uncountable compact subsets of $R^{+}$as $\left\{F_{\alpha}: 1 \leqq \alpha<\Omega\right\}$ where $\Omega$ is the first ordinal having cardinality c. Each $F_{\alpha}$ has cardinality $c$ so that we may inductively choose distinct points $x_{\alpha}$ and $y_{\alpha}$ from the nonempty set $F_{\alpha} \mid\left(Q^{+} \cup\left\{x_{\beta}, y_{\beta}: 1 \leqq \beta<\alpha\right\}\right)$ for each $\alpha<\Omega$. Let $C=Q^{+} \cup\left\{x_{\alpha}: 1 \leqq \alpha<\Omega\right\}$ and $D=Q^{+} \cup\left(R^{+} \mid C\right)$.

EXAMPLE. There is a totally nonmeagre, separable and pseudocomplete metric space $X$ such that $X \times X$ contains a closed subspace homeomorphic to $Q$. Thus $X \times X$ is not totally nonmeagre.

ProOF. We begin by constructing an auxiliary space $Y \subset R$. Let $C$ and $D$ be the subsets of $R^{+}$constructed in Lemma 2 and let $Y=C \cup$ $\{-x: x \in D\}$. Since $R \backslash Y \subset D \cup\{-x: x \in C\}$ is totally imperfect, $Y$ is totally nonmeagre, according to Lemma 1 . Let $\Delta^{\prime}=\{(x,-x): x \in R\}$ be the antidiagonal in $R \times R$. Then the set $K=\Delta^{\prime} \cap(Y \times Y)$ is a closed subspace of $Y \times Y$ and it is easily seen that $K=\{(x,-x): x \in Q\}$ is homeomorphic to $Q$.

To construct the space $X$, we use the standard technique of adding to $Y$ a countable dense set of isolated points. (See, for example, Exercise 14, p. 253 of [2] where such a construction for the space $Q$ is described.) Clearly $X$ can be taken to be a subspace of the Euclidean plane; thus $X$ is separable metrizable. Since $X$ contains a dense set of isolated points, 
$X$ is pseudo-complete. Furthermore, $X$ is totally nonmeagre and $Y$ is a closed subspace of $X$ so that the set $K$, above, is a closed subspace of $X \times X$. Therefore $X \times X$ is not totally nonmeagre.

REMARK. It is clear that the space $Y$ in our example could have been constructed as a subspace of the Cantor set and that the space $X$ could then be obtained by adding countably many isolated points to $Y$, putting one point into each of the open intervals that are removed from the unit interval in the usual construction of the Cantor set. Thus $X$ may be taken to be a subspace of $R$.

\section{REFERENCES}

1. J. M. Aarts and D. J. Lutzer, Pseudo-completeness and the product of Baire spaces, Pacific J. Math. (to appear).

2. N. Bourbaki, Éléments de mathématique. Fasc. VIII. Livre III: Topologie générale, Chap. 9, Actualités Sci. Indust., no. 1045, Hermann, Paris, 1961; English transl., Addison-Wesley, Reading, Mass., 1966. MR 25 \#4480; MR 34 \#5044a.

3. E. Cech, On bicompact spaces, Ann. of Math. (2) 38 (1937), 823-844.

4. Z. Frolík, Locally topologically complete spaces, Dokl. Akad. Nauk SSSR 137 (1961), 790-792 =Soviet Math. Dokl. 2 (1961), 355-357. MR 22 \#9951.

5. K. Kuratowski, Topologie. Vol. 1, PWN, Warsaw, 1958; English transl., Academic Press, New York; PWN, Warsaw, 1966. MR 19, 873; MR 36 \#840.

6. J. C. Oxtoby, Cartesian products of Baire spaces, Fund. Math. $49(1960 / 61)$, 157-166. MR 25 \#4055; errata, MR 26 p. 1453.

Department of Mathematics, Delft Institute of Technology, Delft, The NeTHERLANDS

Department of Mathematics, University of Pittsburgh, Pittsburgh, PennSYLVANIA 15213 